\title{
An empirical, pragmatic approach applying Reflection in Interaction to manual therapy treatments
}

\author{
Neil Tuttle, BSc, MPhil, PhD \\ Griffith University \\ Charles Hazle, BSc, MSc PhD \\ University of Kentucky
}

\begin{abstract}
The majority of literature on decision processes within physiotherapy relates to what can be referred to as "reasoning that results in action" - cognitive processes that determine initial treatment and its modification after reassessment. Particularly expert clinicians also use "reasoning in interaction" including ongoing, interactive processes that occur during the application of treatments. The theory behind the approach discussed in this article can be stated as: If the extent of a deficit (pain or limitation of passive movement) is related to the extent of a patient's limitations (impairments or functional limitations), then 1) a change in the deficit would be expected to be accompanied by a change in the patient's limitations and 2) the relative magnitude of a change in the deficit would be expected to correspond with the relative magnitude of change in the patient's limitations. When applied to manual therapy interventions, the theory suggests that rather than all parameters of a treatment technique being pre-determined, therapists continually adjust their treatment in real time. Adjustments are made in real-time to maximise improvement and are informed by patient response (typically changes in pain or range or stiffness of (passive) movement). Implications for other areas of practice and teaching are discussed.
\end{abstract}




\section{BACKGROUND}

Therapeutic interactions between skilled physiotherapists and patients involve complex, multifaceted, interactive and often creative decision processes. The majority of literature on clinical decision processes relates to a simpler process of what Wainwright et al. (2010), refer to as "reasoning that results in action." In particular, these decision processes relate to cognitively determining a pathological or clinical diagnosis in order to select an initial treatment plan (Roots, Niven, and Moran, 2016). The decision processes within physiotherapy, however, include additional and more complex processes.

A few authors extend their descriptions of clinical decisions beyond the cognitive domain. Forde (1998) considers intuition to be part of the process while Langridge et al. (2015) specifically mentions "gut feeling" as an essential component, particularly among the most highly skilled practitioners. While evidence-guided decisions and systematic approaches to patient care are important, Sizer et al. (2016) offer that patient and clinician interactions are to be the center of clinical reasoning. Recently, Huhn and colleagues (2018) describe the clinical reasoning process as being iterative and adaptive, which is conceptually related to Oberg et al.'s (2015) description of physical therapists using their hands in clinical reasoning to gather and transmit information. Neither group of authors, however, describe the conceptual algorithms clinicians use to process and communicate this information.

Huhn et al. (2018) presented a concept analysis of physiotherapy decision processes within the context of other professions. The nature of decision processes described in different professions varies with the nature of the professions. Diagnostic reasoning is paramount in medicine, the recognition of change in patient symptoms is important in nursing, and narrative or affective 
aspects of reasoning form the focus for psychology. As with all health professions, the majority of literature within physiotherapy describes cognitive diagnostic reasoning or thinking toward a particular clinically relevant label with its associated interventions. The decision processes within physiotherapy, however, include an iterative process that also integrates affective and psychomotor domains. The iterative, real-time decision processes that occur throughout a clinical encounter were referred to by Schon (1987) as "Reflection in Action" and later was perhaps more appropriately referred to as "Reflection in Interaction" (Øberg, Normann, and Gallagher, 2015).

"I begin with the assumption that competent practitioners usually know more than they can say. They exhibit a kind of knowing in practice, most of which is tacit.” (Schon, 1983)

The process of reflection in action is generally considered to be characteristic of more experienced clinicians (Huhn et al., 2018; Roots, Niven, and Moran, 2016; Wainwright, Shepard, Harman and Stephens, 2010). Anecdotally, however, even many novice physiotherapists demonstrate reflection in action by being able to recall occasions during patient interactions when, based on a perceived change during the treatment application, they 'knew' or sensed with confidence a patient was improving during treatment even prior to objectively reassessing their impairments ${ }^{\mathrm{i}}$. An example of the often tacit Reflection in Interaction is that in spite of most schools of manual therapy suggesting that treatment technique and grade are predetermined based on clinical reasoning, the authors have observed clinicians performing moment-to-moment adjustments and refinements of techniques. Such moment-to-moment refinements have been seen to occur not only with passive accessory passive physiological and neural mobilisations, but also McKenzie and Mulligan techniques. Few clinicians or texts, however, describe adjusting 
treatments in real time and those who do fail to describe the processes that inform the decisions behind these adjustments. For example, Maitland suggested his students 'get inside the joint,' but the authors have been unable to find anyone who heard him describe what to do once they got inside the joint.

The aim of the current paper is to provide a theoretical framework and describe the practical application of a process of reasoning in interaction within the psychomotor domain of physiotherapy practice with particular reference to manual therapy for spinal musculoskeletal conditions. The article will 1) describe the theoretical approach, 2) discuss the theory in the context of other approaches to manual therapy, 3) describe the application of the theory to decision making within manual therapy, 4) discuss related concepts, 5) consider the application of the approach to other areas of practice and 6) consider the implications of the approach for teaching and research.

\section{THEORETICAL FRAMEWORK}

Theories within physiotherapy often relate to mechanisms of dysfunction or treatment (Krebs and Harris, 1990). For example, movement dysfunctions of the spine, particularly those featuring mobility loss, are often treated with passive movement and/or active movement, into that previously limited motion. This paper takes a more 'black box' approach in that it does not rely on mechanisms of either patient symptoms or therapeutic interventions. Neither does it presume a cause and effect relationship. The theory is based on a model where, in addition to impairments or functional limitations that are directly measurable, other deficits ${ }^{\mathrm{ii}}$ exist that are related to patient symptoms and are either directly perceivable by the therapist (e.g. tissue response or the extent or quality of either passive or active movements) or able to be verbally communicated to 
the therapist (e.g. extent, quality or location of pain). The theory can then be stated simply as: If the extent of a deficit is related to the extent of a patient's limitations (impairments or functional limitations), then 1) a change in the deficit would be expected to be accompanied by a change in the patient's limitations and 2) the relative magnitude of a change in the deficit would be expected to correspond with the relative magnitude of change in the patient's limitations.

Two important characteristics of theories, in general, are that they should encourage the development of testable hypotheses and such hypotheses should be falsifiable (Krebs and Harris, 1990). In other words, experiments should be able to be designed where some results would be contrary to the theory. The theory described in this paper will be shown to fulfil both of these conditions.

\section{CONTEXT WITHIN OTHER APPROACHES TO MANUAL THERAPY}

"It is not down on any map; true places never are." - Herman Melville, Moby Dick

A range of philosophical approaches underlie different approaches to manual therapy. Interpretation of examination results, adherence to biomechanical principles, assessment of the effects of interventions, timing of the reassessments, and subsequent decision making progressions diverge considerably across these schools of thought (Wise, 2015). Most approaches to manual therapy advocate treatment selection using either a segmental or responder decision making model (Bialosky et al., 2012). Both models consider factors present prior to treatment and select treatment that would be expected to be effective and often target deficits that would be expected to be present. A Patient Response Approach on the other hand is guided by treatments actually being effective. A common and seemingly reasonable version of the patient 
response model is to use reassessment of patient impairments immediately after a treatment application to inform treatment decisions (Tuttle, 2009). The subject of this article could be seen as a subset of the Patient Response Approach where deficits that are actually present are targeted and decisions are informed by changes that actually occur during as well as after an intervention. We will refer to this as a Reflection in Interaction approach. Table 1 provides a comparison between the four approaches described in this manuscript based on the philosophical concepts behind different manual therapy approaches as described by Wise (2015). The authors do not intend to imply that most practitioners utilize a single approach in isolation. Indeed, the skilled practitioner may move fluidly between various models and approaches.

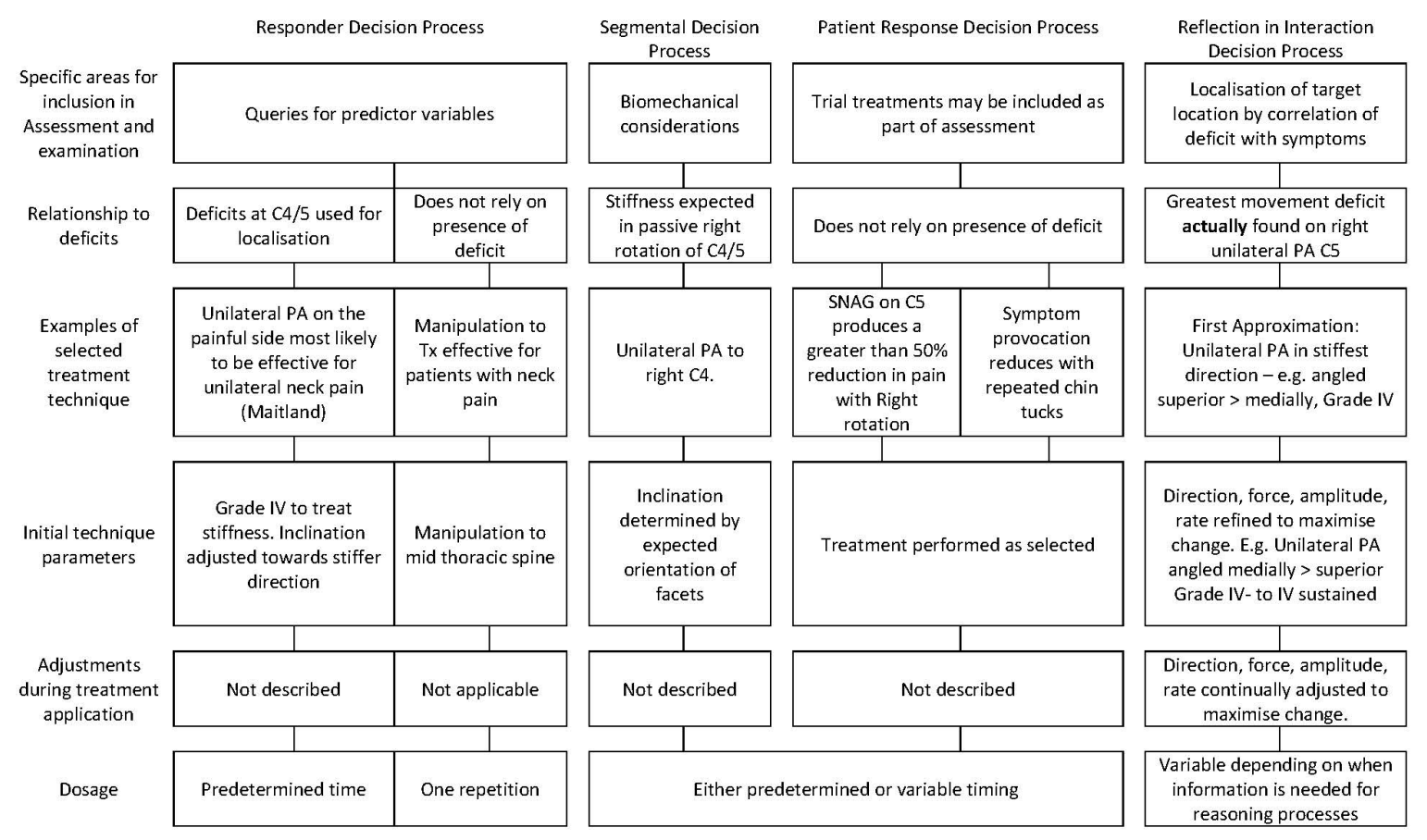

Table 1. Comparison between decision processes for a patient with limited active cervical rotation to the right from a deficit at $\mathrm{C} 4 / 5$. 


\section{$\underline{\text { Segmental decision processes }}$}

Segmental decision processes are typically based on assumed relationships between active movements and passive treatment directions (Bialosky et al., 2012). For example, the Nordic school suggests that a joint should be placed in the loose packed position and mobilisation applied in the direction determined from to the concave/convex rule according to the shape of the joint surfaces and the direction of limited active movement (Wise, 2015). Analysis of the concave/convex rule for the glenohumeral joint yielded conflicting biomechanical results and included a recognition that factors other than joint surface geometry influence kinematics (Brandt, Sole, Krause, and Nel, 2007; Schomacher, 2008; Schomacher, 2009) while for the knee, the accessory glides have been shown to occur in the opposite direction to that determined from the concave/convex rule (Scarvell, et.al. 2019). Other biomechanical approaches suggest patients' limitations of segmental passive movement will correspond with their limitations of active physiological movements. For example, a reduction of active right cervical rotation could result from a deficit in conjoint movements of right rotation and right lateral flexion of $\mathrm{C} 4 / 5$. Treatment would then be applied in the direction of the expected deficit.

Although the simplicity of this type of segmental decision process is attractive, segmental contributions to active movement of the spine are known to be indeterminate (Bogduk and Mercer, 2000). On average, certain patterns of coupled movements occur with particular movements (Cook, Hegedus, Showalter, and Sizer, 2006), but these patterns vary between individuals (Nagamoto et al., 2011) and likely within the same individual (Sizer, Brismee, and Cook, 2007). Furthermore, the movement produced by passive 'accessory' movements such as Postero-Anterior (PA) movements is complex and varies with small changes in the location and direction of the technique (Funabashi et al., 2018). 
In summary, segmental decision processes are used to indicate where deficits would be expected to occur. Treatment is then applied based on these expected deficits even in the absence of the deficits being found to be present. To our knowledge, no studies have either demonstrated deficits actually occurring in the direction predicted by such rules, or that treatment according to these rules improves outcomes.

\section{$\underline{\text { Responder decision processes }}$}

Responder Decision Processes predict treatment effectiveness from patient history and objective assessment. They were perhaps the earliest form of clinical reasoning and often took the form of: technique $\mathrm{X}$ should be used for pattern $\mathrm{Y}$ because it had previously been found to be effective. One form of Responder Decision Process originally described by Maitland suggests that the spinal region and location of pain informs technique selection and the predominance of pain or stiffness informs the grade of movement (Maitland, 1968).

Another example of Responder Decision Processes is the application of developed clinical prediction rules that attempt to determine combinations of patient findings that predict success of a particular treatment. Several clinical prediction rules have been derived pertaining to physiotherapy decision making (Cleland et al., 2005; Flynn et al., 2002; Hicks et al., 2005; Raney et al., 2009; Wainner et al., 2003; Wainner et al., 2005), but only one has a supporting validation study (Childs et al., 2004). Subsequent systematic analysis of the studies leading to the derivation and validation of clinical guidelines has resulted in criticisms of the methodology and interpretation, suggesting only generally weak evidence supports the use of clinical prediction rules (Patel et al., 2013). In summary, the evidence supporting a Responder Decision Process is lacking and has not been shown to produce better outcomes than other decision processes. 


\section{$\underline{\text { Patient Response Approach }}$}

In a Patient Response Approach, the patient's actual - rather than expected - response is used to guide decision making. Common examples of patient response models are reassessing impairments, activity limitations, and/or functional abilities after a treatment as a guide to ongoing decision making (Grieve, 1981; Maitland, Hengeveld, Banks, and English, 2005a; Maitland, Hengeveld, Banks, and English, 2005b). Such within-session changes appear to be reasonable predictors of treatment effectiveness (Tuttle, 2009) and Patient Response Approaches are prevalent throughout the profession (Hahne, Keating, and Wilson, 2004).

Some authors have suggested that decisions on initial treatment selection can also be informed by patients' responses to brief or trial treatments. Improvement following repeated movements of the lumbar spine has been found to predict effective treatment directions for patients with low back pain (May, Runge and Aina, 2018). The use of "treatment direction tests" (Vicenzino, 2004) and trial treatments (Ford, Thompson, and Hahne, 2011) also increase the probability of treatment being effective. Similarly, Mulligan (1993) has suggested that a reduction in pain during active movement while a passive movement is being applied indicates that technique would be expected to be effective. Unlike the previously described Segmental and Responder Decision Processes, there is some evidence that Patient Response Approaches can be reasonable predictors of a future positive response. The Patient Response Approaches described above relate to treatment selection, but do not discuss modification during actual treatment application. In summary, a favourable response to a given treatment is thought to predict future responses to that treatment. 


\section{$\underline{\text { Reflection in Interaction Approach }}$}

The Reflection in Interaction approach is an extension to the Patient Response Approach in two ways. First, there is a 'physical conversation' between the therapist and the patient. Patient responses inform ongoing adjustments to maximise the rate of improvement during (as well as after) a treatment application. Secondly, alterations in any combination of changes in pain and therapist-perceived changes in stiffness, muscle tone, or respiration are used to guide treatment modification.

In its simple form as described in this paper, the approach targets structures and functions. As such it forms part of the 'bio' of a biopsycho-ecological approach (Martinec, 2017). According to WHO classifications, such interventions "are primarily medical or rehabilitative, and attempt to prevent or ameliorate limitations in person or societal level functioning by correcting or modifying intrinsic functions or structures of the body" (WHO, 2005). Although all aspects of a biopsycho-ecological approach are critical, the scope of this article does not allow consideration of other aspects such as exercise, education or psychosocial input, or high velocity techniques.

It is important to reiterate that the physical and verbal conversations between patient and therapist that are being described in this paper form only a small aspect of decision processes that occur within physiotherapy. For example, Ǿberg et al. (2015) elegantly describe a broader embodied enactive reasoning process with "the embodied self [of the therapist] as the source of information, elaboration, and communication in partnership with the patient."

The Reflection in Interaction Approach starts from an initial treatment selection, which can result from any of the decision making processes described above. The theory is that if a deficit 
is related to a patient's impairments, and those impairments are related to the patient's functional limitations, then adjusting treatment parameters to reduce the deficit more quickly would be expected to increase the rate of improvement in the patient's impairments, and thereby in their functional abilities. Conceptually, the Reflection in Interaction Approach can be described as having four cyclical steps; Where, What, How, and Reality Check.

1. Where indicates determining a location that has a motion deficit that is considered to be related to the patient's symptoms (Martinec, 2017). Most commonly, this is a location the clinician considers the source of the patient's symptoms - that is a location where the symptoms can be reproduced. Occasionally, the targeted location can be a contributing factor such as a loss of mobility at a location which is thought to be putting increased load on the source structure, which then becomes symptomatic. Whether a location is, indeed, a contributing factor can be tested in two ways. First, is directing the initial treatment towards the contributing location; an improvement in the patient's impairments on reassessment then supports the hypothesis of that location being a contributor. Secondly, a positive, but transient effect from treating the source location may indicate the need to improve another factor such as another location or movement dysfunction.

2. What results from a more in-depth exploration of the identified deficit and indicates the properties that the clinician hypothesizes can be changed. Historically, treatment decisions have been directed at either pain or stiffness as the predominant factor, and we will consider this dichotomy as a starting point for the Reflection in Interaction Approach.

3. How indicates adjusting the parameters of a treatment technique to determine how it can be performed to maximise change in the target deficit. Importantly, techniques are not considered to have one 'correct' form. Rather, a standard form is considered a first approximation, from which the therapist improvises with intention.

If the intention is to reduce pain, then the idea that a patient's pain should be reduced within and after treatment would seem self-evident. To maximise the within treatment 
change, the therapist may monitor the location, distribution, quality, and/or intensity of pain. In many patients, these pain factors change very rapidly - often within seconds. For some patients, however, such as those with radicular pain, the changes may be much slower. Ways that the techniques can be adjusted to achieve the desired changes include alteration in the direction, force, rate and/or rhythm of the treating movement. An improvement may manifest as a change in the intensity or location of symptoms during treatment or as being able to progress the treating movement before the onset of pain.

When targeting stiffness, the stiffest direction may be a reasonable first approximation of the treatment direction. The stiffest direction, however, will not necessarily produce the most rapid change. Repeated movements in the stiffest (or any) direction in the absence of change suggests the need to alter the direction and/or other parameters of the treatment movement.

4. Reality check: Reassessing impairments. A complete discussion of reassessment is beyond the scope of this paper. One aspect that merits a brief discussion is the timing of reassessment. If the purpose of reassessment is to assist in maximising treatment effectiveness, then reassessment is a problem-solving process intended to answer questions about that patient's responses as they arise rather than something that occurs at set time intervals. If the treatment intention is to increase mobility, then the reassessment may occur either when the mobility of the treatment movement has been perceived to change, or at a time when an effect would be expected to have occurred. Although it is likely that changes in mobility can be detected by the clinician within the application of a treatment technique (see relevant differences perceivable section below), this perception of passive mobility has more potential to be influenced by subconscious bias of the operator than would be the case for changes in active impairments (Tuttle 2009). Reassessment of active impairments, therefore, serves as a reality check. Similarly, if the treatment intention is to reduce pain with a specific passive movement, reassessment might occur once some improvement has occurred in the comfort or ease of the technique or it may occur before any change is noticed in the patient's response to ensure there is no aggravation of symptoms. 
In addition, reassessment may be appropriate when the meaning of a change is uncertain, such as if the both mobility and pain are increasing during treatment. Reassessment between treatment sessions then serves as a further reality check to ensure that withinsession changes in the impairments are, in fact, related to changes in functional abilities and the patient's goals.

After reassessment, the four-step process is repeated considering the additional information of the patient's responses.

\section{ADDITIONAL RELATED CONCEPTS}

There are a several concepts that relate to a Reflection in Interaction Approach including specificity of treatment effects, the ability of clinicians to perceive the relevant differences in mobility, immediate changes predicting longer term changes, and how to record treatments that are continually adjusted rather than being intended to be a precisely repeatable entity. The authors consider it useful to provide a discussion of these concepts to clarify the approach

\section{Specificity of treatment effects}

Although most clinical texts suggest manual therapy treatment should be specific and targeted, the evidence in the literature is mixed. Some studies have found specific treatment to be superior (Chiradejnant, Maher, Latimer, and Stepkovich, 2003; Puentedura et al., 2011; Tuttle, Barrett, and Lasko, 2008) while others have not found differences related to the targeted location, (Aquino et al. 2009; Chiradejnant, Maher, Latimer, and Stepkovich, 2003; Cleland et al., 2009; Kanlayanaphotporn, Chiradejnant, and Vachalathiti, 2009; Schomacher, 2009) or even region (de Oliveira et al., 2013). Many of these studies considered physiological responses to treatment in 
either asymptomatic or symptomatic individuals. The relationship, however, is tenuous between such physiological changes and a potential therapeutic response (Bishop et al., 2017).

Another difficulty in considering the specificity of treatment effects is more subtle and relates to study design. Typical randomised controlled trials (RCTs) compare groups and are, by their nature, incapable of detecting the presence of individual (as distinct from group) responses. A more complete discussion of the difficulties in applying RCTs to clinical practice (Kerry, 2017) and the types of study designs that are necessary for investigating individual responses (Hecksteden et al. 2015; Senn, 2001) can be found elsewhere. Briefly, determining if individuals respond consistently in ways that may be different from group responses requires crossover study designs where individuals receive repeated applications of more than one intervention. The heterogeneity and instability over time of musculoskeletal conditions combined with the heterogeneity of treatments (Snodgrass, Rivett, and Robertson, 2006) and greater variability between than within operators (Seffinger, Naim, Mishra, Adams, et al., 2004) makes the application of such study designs to musculoskeletal conditions particularly difficult. To date, we know of no studies that specifically consider the extent of specificity of individual responses to manual therapy treatments.

In summary, the effectiveness of manual therapy is likely to result from a combination of specific and non-specific factors including mechanical and neurophysiological components (Bialosky et al., 2017). Although clinical experience suggests that individual patients respond consistently to similar interventions and differently to different interventions, there is no evidence that directly evaluates the presence of such patient-by-treatment interactions. 


\section{$\underline{\text { Relevant differences perceivable }}$}

Pain and stiffness are often considered the primary deficits targeted by manual therapy. The ability of patients to perceive and report changes in pain location and intensity occurring during treatment has been clearly demonstrated in the literature on centralisation (May, Runge and Aina, 2018; Yarznbowicz et al., 2018)

The ability of clinicians to perceive differences in stiffness within a treatment session is less clearly established. There is evidence that differences in PA stiffness related to symptoms are greater than the approximately $10 \%$ differences needed to be palpable by manual palpation (Tuttle and Hazle, 2018). Active myofascial trigger points have been found to be $13 \%$ stiffer than corresponding asymptomatic locations and are described as demonstrating a palpable 'release' during treatment (Arokoski et al., 2005). Although not conclusive, the literature therefore suggests that differences in stiffness occurring in clinical practice are likely to be large enough to be perceivable (Tuttle and Hazle, 2018).

\section{Predicting longer term changes}

Both the Patient Response and Reasoning in Interaction Approaches hypothesise that improvements after a given intervention predicts a longer lasting improvement. Questions have been raised about whether short-term effects are appropriate predictors of longer term outcomes (Cook, 2011). Other authors have found that within session changes, particularly in pain and range of movement are reasonable predictors of longer term outcomes (Tuttle, 2009). Specifically, within- and between-session improvements in active range of motion (ROM) have been found to predict between session and end of treatment outcomes for patients with neck pain (Tuttle, 2005; Tuttle, Laakso, and Barrett, 2006) and improvements in pain and ROM predict 
outcomes in patients with lumbar spine symptoms (Cook, Showalter, Kabbaz, and O'Halloran, 2012; Hahne, Keating, and Wilson, 2004). Thus, evidence is lacking of patient responses to predict longer-term outcomes other than that of within and between session changes using manual therapy or exercises guided by centralisation, which have, indeed, been found to be predictive of at least medium-term outcomes.

The usefulness of the Reflection in Interaction Approach described is not, however, conditional on short-term responses being predictive of longer term outcomes. The common application of Patient Response decision processes between-sessions as are used in most approaches to treating musculoskeletal patients would still apply. That is, the relevance of the changes in impairments and/or functional limitations that occur within a treatment session are tested by evaluating changes in functional and participation limitations between sessions.

\section{$\underline{\text { Recording }}$}

Patient notes are a critical aspect of patient care and are both an ethical and legal obligation. (Australian Physiotherapy Association, 2016) Most aspects of patient notes would not be impacted on by using the approaches described in this paper, but recording the treatment warrants some discussion. The Australian Physiotherapy Association (2016) states that "Clinical records must contain sufficient information to allow a physiotherapist of similar skill to continue the treatment of that patient if required." One interpretation of this requirement is that treatments should be able to be duplicated by the same or another therapist as evidenced by the statement; "Standardizing annotation will ensure that future comparisons may be made between studies, reproducibility of techniques between practitioners is guaranteed, and accurate patient records exist for audit purposes" (McDowell, Johnson, and Hetherington, 2014). The type of information 
that is suggested to achieve this intention of reproducibility typically includes: patient position, side, joint or vertebrae, technique, magnitude of force, amplitude, duration, and the use of assistive devices (Hengeveld and Banks, 2014; McDowell, Johnson, and Hetherington, 2014). This type of snapshot approach is fairly uncomplicated when a treatment technique remains constant throughout its application. A slight modification of a snapshot approach can also be used to indicate changes during the treatment application such as a progression through grades of movement (e.g. grade IV- to IV) (Hengeveld and Banks, 2014). When multiple treatment parameters such as the force, rate, and direction are all adjusted during a treatment application as in the Reflection in Interaction Approach, recording the variations in treatment is still possible, but it becomes more difficult to provide sufficient detail and relationships for all parameters of the technique that would be necessary for the technique to be replicated.

If the same therapist is reproducing the same technique, the repeatability of their applied forces may be good to excellent (Snodgrass, Rivett, Robertson, and Stojanovski, 2010). If a different operator is performing the technique, however, regardless of how precisely treatment parameters are recorded, there are difficulties in the idea that manual treatments can be accurately reproduced. The force and direction with the same technique and grade varies greatly (Snodgrass, Rivett, and Robertson, 2006). As an example, there are significant differences between the mean forces applied by different students or experienced therapists performing the same technique on the same location which can vary by at least a factor of four (Snodgrass, Rivett, Robertson, and Stojanovski, 2010). In other words, if using grades according to the Maitland approach of I to IV, the amount of force applied by some students or therapists for a grade I movement would be greater than the force used by others with similar experience to 
produce a grade IV (Snodgrass, Rivett, and Robertson, 2007). The difficulty is further increased by 1) the direction of applied force with the same technique varies between therapists (Snodgrass, Rivett, and Roberston, 2009); 2) even if the direction of the technique remains consistent, differences in the way it is performed create quite different movements of the underlying vertebrae (Funabashi et al., 2018); and 3) clinicians are unable to consistently locate a particular spinal location. For example, even the spinous process of $\mathrm{C} 7$ which would be expected to be amongst the easiest to locate can only be correctly located about half of the time (Póvoa, Ferreira, Zanier, and Silva, 2018). Although it is useful to provide a record of what treatment is performed, regardless of whether treatments are applied as a fixed entity or part of an adaptive process, it is probably unreasonable to expect treatments to be repeatable by another therapist based on these records.

A narrative approach presents an alternative way of documenting patient care that is still consistent with the intention that a similarly skilled therapist would be able to continue the care (Australian Physiotherapy Association, 2016): "The narrative is both a picture of an unfolding story within a single occasion of care as well as the connectedness of the occasions over time." A narrative approach to record keeping might indicate 1) a hypothesised relationship between localised deficits, patient impairments and limitations in function or participation; 2) what aspects of the deficits the treatments are expected to improve; 3 ) techniques used and what parameters were varied; 4) patient response during and after treatment; and 5) implications and plan for further management. 
The intention of record keeping to enable a therapist to effectively continue treatment can be achieved by a variety of approaches to record keeping. Regardless of whether duplicating a technique or treatment is necessary or desirable, it is unlikely that any of the available approaches to record keeping would be sufficient by itself to enable a clinician to duplicate a technique or treatment. Narrative approaches on the other hand would appear to be able to adequately describe more complex decision processes as well as fulfilling the legal and functional requirements of patient records.

\section{DISCUSSION}

A pragmatic, empirical approach to Reflection in Interaction is presented in relation to manual therapy treatments. The approach starts with identification of a deficit in pain and/or passive range of movement that is perceivable by the therapist that can be hypothesised to be related to the patient's impairments. Treatment is selected and modified to maximise real-time improvement in the deficit that is being targeted. Reassessment of the patient's impairments then serves to confirm or refute the therapist's hypothesis of the relationship between the therapistperceivable deficits and the patient impairments.

\section{$\underline{\text { Mechanism agnostic }}$}

Although musculoskeletal problems can result in pain and reduction of ROM, the mechanisms that produce these impairments are not well understood (Bialosky et al., 2017). The Nordic approach is based on biomechanical relationships consistent with structural factors limiting movement and treatment altering those structures, the Mulligan approach suggests 'positional faults' are responsible for reduced ROM and are improved with treatment while other authors suggest reduced ROM and treatment effects result from muscle responses to neurophysiological 
factors (Wise, 2015). A patient who has central pain sensitisation without a known physiological basis may still improve on a moment to moment basis with repetition of gentle active or passive movements. We are suggesting an empirically testable approach that can be applied regardless of the mechanisms of either movement limitation or treatment effects. Hypotheses are generated that are specific for a given patient at a given time point and take the form: a change in a particular deficit during treatment is expected to be followed by changes in impairments after the treatment. Each hypothesis is, therefore, both testable and falsifiable (the hypothesised change either does or does not occur) but is not dependent on either cause of the deficit or mechanism of treatment.

Each hypothesis is provisional since continued use of deficits as predictors is predicated on the presence of a continued relationship between changes in the deficits during treatment, changes in impairments on post-treatment reassessment, and persistent changes in impairments and functional abilities between treatment sessions. For example, a patient with a radiculopathy may have a lack of or limited response during treatment, but a significant improvement afterwards between treatments. Alternatively, for a patient with central sensitisation or unspecified injuries after a vehicle accident either no change or an immediate improvement during treatment be transient or followed by a latent increase in symptoms. The hypothesis of a relationship between immediate and longer term responses would thus be falsified and not be continued. In both of these instances, the therapist's response would be the same as if another segmental or patient response approach was being applied and treatment modification were based on reassessment of impairments following treatment. That is, the treatment would be modified or abandoned. 


\section{$\underline{\text { Implications for practice }}$}

The approach has been primarily described in relation to passive movements of the spine such as Postero-Anterior movements (PAs). The concepts, however, of adjusting multiple parameters of a treatment to maximise change in the targeted deficit are equally applicable to a wide range of treatment options. The force, rate and direction of passive mobilisation of physiological movements can be adjusted in in a similar way. If a patient is doing active stretching to increase mobility, whether targeting contractile or non-contractile structures, and if the patient understands the intention, the exercise technique can be adjusted to maximise the change. When prescribing exercises such as controlled loading for an Achilles tendinopathy, rather than a predetermined load (typically either pain-free movement or load to tolerance), the load can be adjusted to maximise symptom change during repeated movements. Similar approaches have also been described in relation to patients with neurological conditions (Øberg, Normann, and Gallagher, 2015).

\section{$\underline{\text { Implications for teaching and research }}$}

Although many experienced clinicians appear to use an approach similar to what is being described in this paper, the approach does not appear to be commonly taught to either entry-level or postgraduate students. Perhaps segmental or algorithm-based reasoning processes are considered conceptually more accessible. Our experience is that the approach being proposed is well-tolerated by students and may, in fact, be conceptually simpler than the various segmental or algorithm-based approaches. For example, a recent cohort of students undertook a peripheral musculoskeletal course which was taught according to a Nordic rule based approach. The next semester their spinal course was taught according to the Reflection in Interaction Approach 
described in this paper. Many students indicated that they had found the rule-based approach confusing and the Reflection in Interaction Approach conceptually simpler and more accessible even if they could not yet perceive the level of differences in spinal mobility that would be needed to fully apply the principles. More detailed descriptions of aspects of the approaches used in teaching manual therapy skills are provided elsewhere (Tuttle and Bialocerkowski 2017; Tuttle, Evans, Bialocerkowski, 2015; Tuttle and Hazle, 2018).

The question of what level of complexity is accessible to students presents an on-going challenge to educators. Rule-based approaches can be useful to provide a first approximation or be used when student skills are not sufficient to detect moment-to-moment changes. The Reflection in Interaction decision process may be viewed as a higher level skill toward which students and novice practitioners can aspire to during their development. In use of this model, the application of cognitive and sensorimotor processes are conditionalized based upon patient specific circumstances. Decisions do not follow a prescriptive structure based on examination findings or other clinical features, but rather are informed by the overall context of that patient.

Even beginning students appear to have the cognitive and perceptual capacity to be responsive to changes in their patients' condition. Further, we propose that educators can assist their students' development by providing conceptual frameworks to interactively integrate cognitive and psychomotor domains of learning.

There are several areas of research that would be useful in relation to the Reflection in Interaction Approach. Qualitative investigations would be useful 1) to determine how 
experienced clinicians process the type of decisions that are being described and 2) how patients perceive and respond to the interactivity of the process. Eventually a classical group RCT would be appropriate comparing outcomes from proscriptive and interactive decision processes. Prior to such an RCT, however, other study designs such as aggregated $\mathrm{N}$ of 1 trials would help to address the validity of some of the related concepts such as the presence of individual as distinct from group responses and patient by treatment interactions.

\section{DECLARATIONS}

No funding was received for this manuscript. The authors report no conflicts of interest.

\section{$\underline{\text { SUMMARY AND CONCLUSIONS }}$}

Frequently, the reasoning underlying treatment selection is contingent on deficits that would be expected to occur and treatments that would be expected to be effective. The Reflection in Interaction Approach presented here starts with determining deficits that actually exist and uses moment-to-moment changes in those deficits that actually occur to guide clinical decision processes. Hypotheses that are testable and falsifiable follow directly from the theory. For a particular patient, the hypotheses take the form of: if deficit $\mathrm{X}$ improves during a treatment application, then impairments $\mathrm{Y}$ or $\mathrm{Z}$ would be expected to be improved when reassessed immediately after the treatment. The normal process of reassessing impairments, functional and participation limitations within and between treatment sessions can then be used to ensure the relevance of within-session improvement in that impairment for that patient achieving their goals. 
Our unproven opinion is that this approach not only improves patient outcomes, but also makes the process of clinical reasoning more interesting and engaging, albeit less predictable, for both novice and advanced clinicians. The authors are in the process of conducting clinical trials using multiple single case study designs to investigate the presence of patient by treatment interactions and to evaluate the effectiveness of the approach described in this paper.

\section{References}

Aquino RL, Caires PM, Furtado FC, Loureiro AV, Ferreira PH and Ferreira ML 2009 Applying joint mobilization at different cervical vertebral levels does not influence immediate pain reduction in patients with chronic neck pain: A randomized clinical trial. Journal of Manipulative and Physiological Therapeutics. 17: 95-100.

Arokoski JP, Surakka J, Ojala T, Kolari P, Jurvelin JS 2005 Feasibility of the use of a novel soft tissue stiffness meter. Physiological Measurement 26: 215.

Austrailian Physiotherapy Association 2016 Guidelines for writing clinical notes. https://www.physiotherapy.asn.au/DocumentsFolder/Advocacy/APA_Writing\%20Clinical\%20N otes\%20Guidelines\%202018_v4\%20FA.pdf.

Bialosky JE, Beneciuk JM, Bishop MD, Coronado RA, Penza CW, Simon CB, George SZ 2017 Unraveling the mechanisms of manual therapy: Modeling an approach. Journal of Orthopaedic and Sports Physical Therapy: 1-31.

Bialosky JE, Simon CB, Bishop MD, George SZ 2012 Basis for spinal manipulative therapy: A physical therapist perspective. Journal of Electromyography and Kinesiology 22: 643-647.

Bishop MD, Bialosky JE, Penza CW, Beneciuk JM, Alappattu MJ 2017 The influence of clinical equipoise and patient preferences on outcomes of conservative manual interventions for spinal pain: An experimental study. Journal of Pain Research 10: 965-972.

Bogduk N, Mercer S 2000 Biomechanics of the cervical spine. I: Normal kinematics. Clinical Biomechanics 15: 633-648.

Brandt, C, Sole G, Krause MW, Nel M 2007 An evidence-based review on the validity of the Kaltenborn rule as applied to the glenohumeral joint. Manual Therapy 12: 3-11. 
Childs JD, Fritz JM, Flynn TW, Irrgang JJ, Johnson KK, Majkowski GR and Delitto A 2004 A clinical prediction rule to identify patients with low back pain most likely to benefit from spinal manipulation: A validation study. Annals of Internal Medicine 141: 920-928.

Chiradejnant A, Maher CG, Latimer J and Stepkovitch N 2003 Efficacy of "therapist-selected" versus "randomly selected" mobilisation techniques for the treatment of low back pain: A randomised controlled trial. Australian Journal of Physiotherapy 49: 233-241.

Cleland JA, Fritz JM, Kulig K, Davenport TE, Eberhart S, Magel J and Childs JD 2009 Comparison of the effectiveness of three manual physical therapy techniques in a subgroup of patients with low back pain who satisfy a clinical prediction rule: A randomized clinical trial. Spine (Phila Pa 1976) 34: 2720-2729.

Cook C 2011 Immediate effects from manual therapy: Much ado about nothing? Journal of Manual \& Manipulative Therapy 19: 3-4.

Cook C, Hegedus E, Showalter C and Sizer Jr P 2006 Coupling behavior of the cervical spine: A systematic review of the literature. Journal of Manipulative and Physiological Therapeutics 29: 570.

Cook CE, Showalter C, Kabbaz V and O'Halloran B 2012 Can a within/between-session change in pain during reassessment predict outcome using a manual therapy intervention in patients with mechanical low back pain? Manual Therapy 17: 325-329.

de Oliveira RF, Liebano RE, Costa LdCM, Rissato LL and Costa LOP 2013 Immediate effects of region-specific and non-region-specific spinal manipulative therapy in patients with chronic low back pain: A randomized controlled trial. Physical Therapy 93: 748-756.

Flynn T, Fritz J, Whitman J, Wainner R, Magel J, Rendeiro D, Butler B, Garber M and Allison S 2002 A clinical prediction rule for classifying patients with low back pain who demonstrate short-term improvement with spinal manipulation. Spine 27: 2835-2843.

Ford JJ, Thompson SL and Hahne AJ 2011 A classification and treatment protocol for low back disorders: Part 1-specific manual therapy. Physical Therapy Reviews 16: 168-177.

Forde R. 1998 Competing conceptions of diagnostic reasoning--is there a way out? Theoretical Medicine and Bioethics 19: 59-72.

Funabashi M, Nougarou F, Descarreaux M, Prasad N and Kawchuk GN 2018 Does the application site of spinal manipulative therapy alter spinal tissues loading? Spine Journal 18:1041-1052.

Grieve GP 1981. Common vertebral joint problems. Melbourne: Churchill Livingstone. 
Hahne AJ, Keating JL and Wilson SC 2004 Do within-session changes in pain intensity and range of motion predict between-session changes in patients with low back pain? Australian Journal of Physiotherapy 50: 17-23.

Haneline M, Cooperstein R, Young M, Birkeland K. 2009 An annotated bibliography of spinal motion palpation reliability studies. Joural Canadian Chiropractic Association. 53:40-58.

Hecksteden A, Kraushaar J, Scharhag-Rosenberger F, Theisen D, Senn S and Meyer T 2015 Individual response to exercise training - a statistical perspective. Journal of Applied Physiology 118: 1450-1459.

Hengeveld E and Banks K 2014 Maitland's vertebral manipulation: Management of neuromusculoskeletal disorders. Volume 1. Edinburgh: Elsevier.

Hicks GE, Fritz JM, Delitto A and McGill SM 2005 Preliminary development of a clinical prediction rule for determining which patients with low back pain will respond to a stabilization exercise program. Archives of Physical Medicine and Rehabilitation 86: 1753-1762.

Huhn K, Gilliland SJ, Black LL, Wainwright SF and Christensen N 2018 Clinical reasoning in physical therapy: A concept analysis. Physical Therapy. 99: 440-56.

Huijbregts PA 2002 Spinal motion palpation: a review of reliability studies. Journal of Manual and Manipulative Therapy. 10: 24-39.

Kanlayanaphotporn R, Chiradejnant A and Vachalathiti R 2009 The immediate effects of mobilization technique on pain and range of motion in patients presenting with unilateral neck pain: A randomized controlled trial. Archives of Physical Medicine and Rehabilitation 90: 187192.

Kerry R 2017 Expanding our perspectives on research in musculoskeletal science and practice. Musculoskeletal Science and Practice 32: 114-119.

Krebs DE and Harris SR 1990 Elements of theory presentations in physical therapy. Physiotherapy Theory and Practice 6: 57-63.

Langridge N, Roberts L and Pope C 2015 The clinical reasoning processes of extended scope physiotherapists assessing patients with low back pain. Manual Therapy 20: 745-750.

Maitland G, Hengeveld E, Banks K and English K 2005a Maitland's peripheral manipulation. Edinburgh, New York: Elsevier/Butterworth Heinemann.

Maitland GD 1968 Vertebral manipulation. London: Butterworths.

Maitland GD, Hengeveld E, Banks K and English K 2005b Maitland's vertebral manipulation. Edinburgh, New York: Elsevier/Butterworth Heinemann. 
Martinec R 2017 Physiotherapy and rehabilitation: New paradigms and challenges. Journal of Physiotherapy and Rehabilitation 2: 1035-1045.

May S, Runge N, and Aina A 2018 Centralization and directional preference: An updated systematic review with synthesis of previous evidence. Musculoskeletal Science and Practice 38: 53-62.

McDowell JM, Johnson GM and Hetherington BH 2014 Mulligan concept manual therapy: Standardizing annotation. Manual Therapy 19: 499-503.

Mulligan BR 1993 Mobilisations with movement (mwm's). Journal of Manual \& Manipulative Therapy 1: 154-156.

Nagamoto Y, Ishii T, Sakaura H, Iwasaki M, Moritomo H, Kashii M, Hattori T, Yoshikawa H and Sugamoto K 2011 In vivo three-dimensional kinematics of the cervical spine during head rotation in patients with cervical spondylosis. Spine (Phila Pa 1976) 36: 778-783.

Øberg GK, Normann B and Gallagher S 2015 Embodied-enactive clinical reasoning in physical therapy. Physiotherapy Theory and Practice 31: 244-252.

Patel S, Friede T, Froud R, Evans DW and Underwood M 2013 Systematic review of randomized controlled trials of clinical prediction rules for physical therapy in low back pain. Spine (Phila Pa 1976) 38: 762-769.

Puentedura EJ, Landers MR, Cleland JA, Mintken PE, Huijbregts P and Fernandez-de-Las-Penas C 2011 Thoracic spine thrust manipulation versus cervical spine thrust manipulation in patients with acute neck pain: A randomized clinical trial. Journal of Orthopaedic and Sports Physical Therapy.

Raney NH, Petersen EJ, Smith TA, Cowan JE, Rendeiro DG, Deyle GD and Childs JD 2009 Development of a clinical prediction rule to identify patients with neck pain likely to benefit from cervical traction and exercise. European Spine Journal 18: 382-391.

Roots SA, Niven E and Moran RW 2016 Osteopaths' clinical reasoning during consultation with patients experiencing acute low back pain: A qualitative case study approach International. Journal of Osteopathic Medicine 19: 20-34.

Scarvell JM, Hribar N, Galvin CR, Pickering MR, Perriman DM, Lynch, JT, and Smith PN 2019. Analysis of Kneeling by Medical Imaging Shows the Femur Moves Back to the Posterior Rim of the Tibial Plateau, Prompting Review of the Concave-Convex Rule. Physical Therapy, 99: 311-318.

Schomacher J 2009 The convex-concave rule and the lever law. Manual Therapy 14: 579-582. 
Schomacher J 2009 The effect of an analgesic mobilization technique when applied at symptomatic or asymptomatic levels of the cervical spine in subjects with neck pain: A randomized controlled trial. Journal of Manual \& Manipulative Therapy 17: 101-108.

Schön DA 1987. Educating the reflective practitioner: Toward a new design for teaching and learning in the professions. San Francisco: Jossey-Bass.

Seffinger MA, Najm WI, Mishra SI, Adams A, Dickerson VM, Murphy LS, et al. 2004 Reliability of spinal palpation for diagnosis of back and neck pain: a systematic review of the literature. Spine. 29: E413-25.Senn S 2001 Individual therapy: New dawn or false dawn? Drug Information Journal 35: 1479-1494.

Sizer PS, Jr., Brismee JM and Cook C 2007 Coupling behavior of the thoracic spine: A systematic review of the literature. Journal of Manipulative and Physiological Therapeutics 30: 390-399.

Sizer PS Jr, Mauri MV, Learman K, Jones C, Gill N, Showalter CR and Brismee JM 2016 Should evidence or sound clinical reasoning dictate patient care? Journal of Manual and Manipulative Therapy 24: 117-119.

Snodgrass SJ, Rivett DA and Robertson VJ 2006 Manual forces applied during posterior-toanterior spinal mobilization: A review of the evidence. Journal of Manipulative and Physiological Therapeutics 29: 316-329.

Snodgrass SJ, Rivett DA and Robertson VJ 2007 Manual forces applied during cervical mobilization. Journal of Manipulative and Physiological Therapeutics 30: 17-25.

Snodgrass SJ, Rivett DA, Robertson VJ and Stojanovski E 2009 Forces applied to the cervical spine during posteroanterior mobilization. Journal of Manipulative and Physiological Therapeutics 32: 72-83.

Snodgrass SJ, Rivett DA, Robertson VJ and Stojanovski E 2010 A comparison of cervical spine mobilization forces applied by experienced and novice physiotherapists. Journal of Orthopaedic and Sports Physical Therapy 40: 392-401.

Tuttle N 2005 Do changes within a manual therapy treatment session predict between-session changes for patients with cervical spine pain? Australian Journal of Physiotherapy 51: 43-48.

Tuttle N 2009 Is it reasonable to use an individual patient's progress after treatment as a guide to ongoing clinical reasoning? Journal of Manipulative and Physiological Therapeutics 32: 396403.

Tuttle N, Barrett R and Laakso L 2008 Relation between changes in posteroanterior stiffness and active range of movement of the cervical spine following manual therapy treatment. Spine (Phila Pa 1976) 33: E673-E679. 
Tuttle N and Bialocerkowski A 2017 Developing student skills to actively engage in feedback conversations: A pilot study. Internet Journal of Allied Health Sciences and Practice 15: 10.

Tuttle N, Evans K and Bialocerkowski A 2015. Integrating science, technology, and human interaction in teaching musculoskeletal physiotherapy. Presented at World Confederation of Physical Therapy, Singapore.

Tuttle N and Hazle C 2018 Spinal PA movements behave 'as if' there are limitations of local segmental mobility and are large enough to be perceivable by manual palpation: A synthesis of the literature. Musculoskeletal Science and Practice 36: 25-31.

Tuttle N, Laakso L and Barrett R 2006 Change in impairments in the first two treatments predicts outcome in impairments, but not in activity limitations, in subacute neck pain: An observational study. Australian Journal of Physiotherapy 52: 281-285.

Vicenzino B 2004 Foot orthotics in the treatment of lower limb conditions: A musculoskeletal physiotherapy perspective. Manual Therapy 9: 185-196.

Wainner RS, Fritz JM, Irrgang JJ, Boninger ML, Delitto A and Allison S 2003 Reliability and diagnostic accuracy of the clinical examination and patient self-report measures for cervical radiculopathy. Spine (Phila Pa 1976) 28: 52-62.

Wainner RS, Fritz JM, Irrgang JJ, Delitto A, Allison S and Boninger ML 2005 Development of a clinical prediction rule for the diagnosis of carpal tunnel syndrome. Archives of Physical Medicine and Rehabilitation 86: 609-618.

Wainwright SF, Shepard KF, Harman LB and Stephens J 2010 Novice and experienced physical therapist clinicians: A comparison of how reflection is used to inform the clinical decisionmaking process. Physical Therapy 90: 75-88.

Wise CH 2015. Orthopaedic manual physical therapy from art to evidence. Philadelphia: FA Davis.

World Health Organization 2005 Towards a common language for functioning, disability and health: International Classification of Functioning, Disability and Health 2002 Available at: www3. who. int/icf/beginners/bg. pdf.

Yarznbowicz R, Tao M, Owens A, Wlodarski M Dolutan J 2018 Pain pattern classification and directional preference are associated with clinical outcomes for patients with low back pain. Journal of Manual \& Manipulative Therapy 26: 18-24.

Huhn, K, SJ Gilliland, LL Black, SF Wainwright and N Christensen 2018 Clinical Reasoning in Physical Therapy: A Concept Analysis. Physical Therapy 
Wainwright, SF, KF Shepard, LB Harman and J Stephens 2010 Novice and experienced physical therapist clinicians: a comparison of how reflection is used to inform the clinical decisionmaking process. Physical Therapy 90: 75-88

${ }^{\mathrm{i}}$ Impairments will be used in accordance with the International Classification Framework and will generally indicate pain or limitation of active movement or functional activity, but can refer to "any loss or abnormality of psychological, physiological or anatomical structure or function. .. In principle, impairments represent disturbances at the organ level." (http://www.who.int/kobe_centre/ageing/ahp_vol5_glossary.pdf?ua=1)

${ }^{\text {ii }}$ Deficit here is will be considered to have a somewhat different meaning than that used in ICF. In this case deficit will be used to indicate the specific limitations within a particular structure or motion segment that a person actually has. For example, a patient with symptoms on right rotation thought to be coming from $\mathrm{C} 4 / 5$ might be expected to have a limitation of passive right rotation and right lateral flexion at $\mathrm{C} 4 / 5$ (and/or of unilateral PA to $\mathrm{C} 4$ on the right). The deficit that is actually found, however, might be in entirely different directions. 